\title{
Telorragia en el lactante por ectasia ductal. Consideraciones acerca de dos casos
}

Bloody nipple discharge. Duct ectasia

\author{
Dr. Pablo Nicolás Affranchino ${ }^{a}$, Dr. Juan Pablo Oglietti ${ }^{a}$ Dr. Diego Amoedo y Dra. Mariana Nastri ${ }^{a}$
}

\begin{abstract}
RESUMEN
La descarga de sangre a través del pezón (telorragia) es un síntoma muy infrecuente en pediatría. Si bien genera gran preocupación y alarma por su equivocada asociación con el carcinoma de pacientes adultos, en los niños se asocia, en la mayoría de los casos, con una ectasia ductal benigna. Hasta el momento, sólo existen informes o series de casos y, aunque se proponen diferentes teorías, la causa no se conoce con exactitud. Se presentan dos casos con características clínicas y evolución muy similar, en los que el sangrado se resolvió en forma espontánea, uno en cuatro y el otro en seis semanas.

Se realiza una revisión del tema y, dado que se trata de un proceso benigno y autolimitado, se sugiere, en ausencia de otros hallazgos en el examen físico, realizar estudios mínimamente invasivos, adoptar una conducta expectante y tranquilizar a la familia en la espera de una resolución espontánea.

Palabras clave: telorragia, ectasia ductal, sangrado a través del pezón.
\end{abstract}

\section{SUMMARY}

Bloody discharge from the nipple (thelorrhagia) is a rare symptom in childhood and is a cause of great concern due to the mistaken association with carcinoma in adults. However, in children thelorrhagia is due to benign mammary duct ectasia in the majority of cases. Up to date, only isolated case reports or case series have been published and, although different theories have been postulated, no definite etiology has been identified. We report two cases with similar clinical features and outcome, in whom bleeding resolved spontaneously 4 and 6 weeks after onset.

We present a review of the literature. Based on the benign and self-limiting process and in the absence of other findings on physical examination, we suggest a watch-and-wait approach with minimally invasive studies and reassurance and support for the family as most cases resolve spontaneously.

Key words: thelorrhagia, duct ectasia, bloody nipple discharge.

http:/ /dx.doi.org/10.5546/aap.2013.e152

a. Clínica Pediátrica. Área ambulatoria.

Servicio de Bajo Riesgo. Hospital de Pediatría

"Prof. Dr. Juan P. Garrahan".

Correspondencia:

Dr. Pablo Nicolás Affranchino: affranchino@gmail.com

Conflicto de intereses: Ninguno que declarar.

Recibido: 2-7-2013

Aceptado: 25-9-2013

\section{INTRODUCCIÓN}

La telorragia o sangrado a través del pezón es un síntoma de observación poco habitual en la práctica pediátrica. Genera gran preocupación y sorpresa, tanto en la familia del paciente como en el equipo médico tratante. Esta excesiva preocupación se debe a su equivocada asociación con el carcinoma de mama en los pacientes adultos.

Existen muy pocos casos publicados hasta el momento y no se describió ningún proceso maligno en menores de 3 años; por el contrario, se trata de una entidad benigna, autolimitada y aunque la etiología no se conoce con precisión, se asocia en la mayoría de los casos con ectasia ductal benigna. ${ }^{1-5}$

Presentamos dos casos clínicos de lactantes con sangrado por el pezón con evolución típica de ectasia ductal benigna.

\section{CASOS CLÍNICOS}

\section{Paciente 1}

Se trata de un varón de 4 meses que presenta una secreción hemorrágica por ambos pezones, de dos semanas de evolución.

Su madre describe la secreción como espontánea, intermitente y evidenciada por la aparición de "manchas en la ropa".

Es un paciente antes sano, sin antecedentes perinatológicos de importancia, alimentado con leche materna en forma exclusiva, sin antecedentes de traumatismos ni de coagulopatías en su familia. Del interrogatorio no surge como antecedente la ingesta de fármacos.

En el examen físico se observa a un lactante en excelente estado general, sonriente y afebril. En la inspección presenta un pequeño sangrado espontáneo en el área mamilar derecha (Figura 1).

No se palpa aumento de tamaño de las glándulas mamarias. No presenta adenopatías. Genitales masculinos normales. Sin otras manifestaciones hemorrágicas.

Se realizan exámenes de laboratorio, ecografía mamaria y se toma muestra de la secreción para anatomía patológica y cultivo. 
Los análisis de sangre revelan resultados dentro de límites normales: prolactina $15,3 \mathrm{mg} /$ $\mathrm{ml}$, TSH 1,01 $\mu \mathrm{g} / \mathrm{ml}, \mathrm{T}_{4}$ libre $1,20 \mu \mathrm{g} / \mathrm{ml}, \mathrm{T}_{4} 10$ $\mu \mathrm{g} / \mathrm{ml}, \mathrm{T}_{3} 2,16 \mu \mathrm{g} / \mathrm{ml}$, estradiol $\leq 9 \mathrm{pg} / \mathrm{ml}$, Quick $94 \%$, TTPa $38 \mathrm{~s}$, glóbulos blancos $6780 / \mathrm{mm}^{3}(28-$ 62-9-1-0), Hb 10,4 g/dl, plaquetas 380 000/ $\mathrm{mm}^{3}$.

Ecografía mamaria (Figura 2): ambas glándulas mamarias discretamente aumentadas de tamaño, de ecoestructura finamente heterogénea, sin dilataciones ductales. No presenta hallazgos patológicos en la evaluación Doppler color. Sin colecciones.

Informe de anatomía patológica: tres extendidos citológicos teñidos con técnica de hematoxilina y eosina donde se observan hematíes y aislados leucocitos. Sin presencia de células atípicas ni de proceso inflamatorio agudo.

Cultivo de secreción: negativo.

FigURA 1. Secreción hemorrágica espontanea en pezón derecho

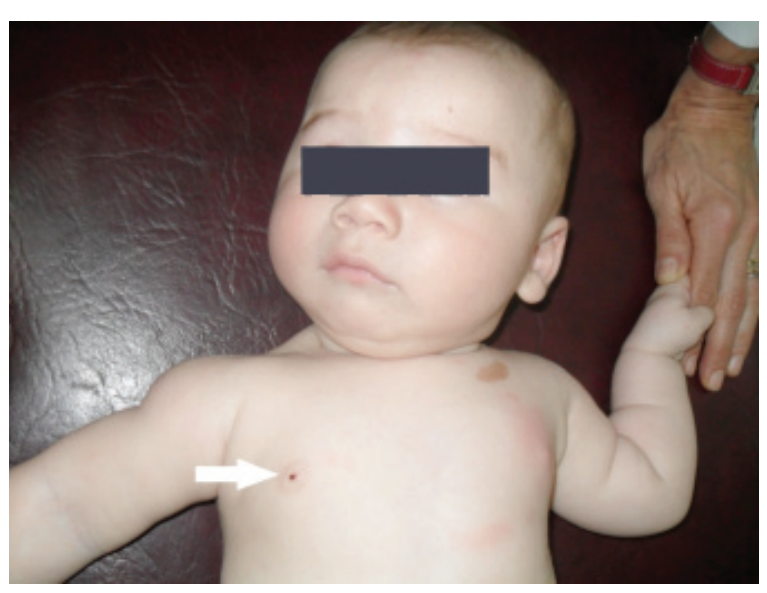

FIGURA 2. Ecografía mamaria normal

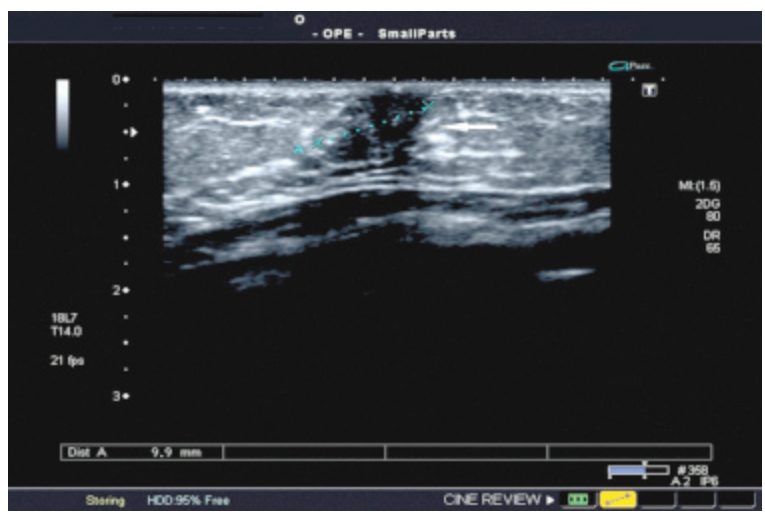

Con los estudios anteriormente citados se asume el cuadro como probable ectasia ductal benigna y se toma una conducta expectante. La telorragia cede de manera espontánea al cabo de 6 semanas.

\section{Paciente 2}

Corresponde a un varón de 5 meses que presenta una secreción hemorrágica del pezón izquierdo, de 3 a 4 días de evolución. Sus padres concurren con gran preocupación después de haber realizado consultas previas en otros centros.

Se trata de un paciente antes sano, sin antecedentes perinatológicos de importancia, alimentado con leche materna y que recibe complemento con fórmula de inicio. No ha sufrido traumatismos, no recibió fármacos ni tampoco hay antecedentes de coagulopatías en su familia.

El estado general es excelente y, como en el caso anterior, no hay datos semiológicos relevantes más allá de la secreción descrita. Ver video en versión electrónica: se observa la típica descarga de sangre por el pezón, tras ejercer una presión suave.

En esta oportunidad se realiza una ecografía mamaria y extendido del material para anatomía patológica, pero no se solicitan análisis de laboratorio por no observar aumento del tejido mamario.

Ecografía mamaria: se explora con transductor de alta frecuencia la región mamaria, observándose dilatación arrosariada de los conductos canaliculares, algunos con contenido heterogéneo y sin evidencia de vascularización, hallazgos compatibles con ectasia ductal mamaría (Figura 3).

\section{FIgURA 3. Ecografía mamaria con ectasia ductal}

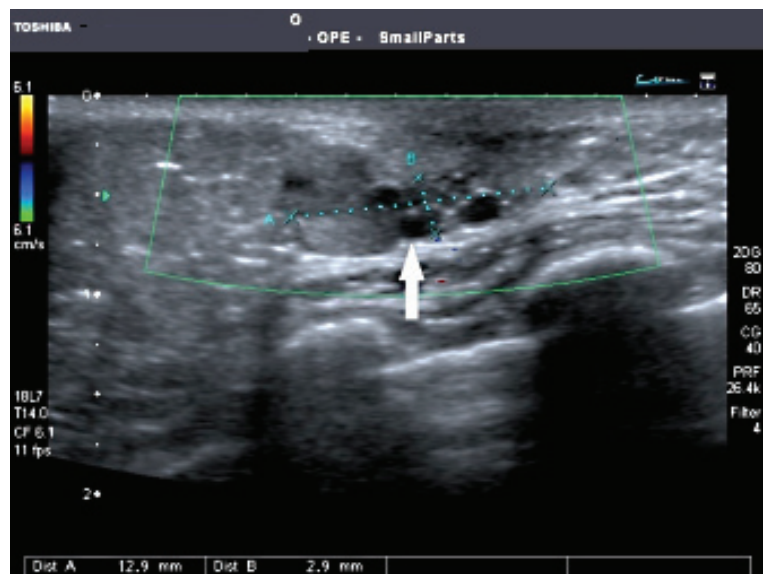


Informe de anatomía patológica: dos extendidos citológicos teñidos con técnica de hematoxilina y eosina que muestran abundantes hematíes, elementos polinucleares y mononucleares (elementos figurados de la sangre). Presencia de macrófagos espumosos y células pavimentosas descamadas aisladas con disqueratosis. No se observan células atípicas ni de proceso inflamatorio agudo.

Asumiéndose el caso como ectasia ductal benigna, se toma una conducta expectante y se comprueba la desaparición del signo a las cuatro semanas de su inicio.

\section{DISCUSIÓN}

Las mamas sufren diversas variaciones desde el nacimiento hasta la adquisición de su forma y volumen al final de la adolescencia.

Al nacer, debido al impacto de las hormonas maternas y placentarias, es frecuente que los neonatos presenten un botón mamario unilateral o bilateral. Esta situación puede mantenerse durante varias semanas, aunque debería involucionar conforme pasen los primeros 3 a 6 meses. Puede producirse, también, secreción de calostro (leche de brujas) y, en las niñas, hiperplasia del endometrio con su posterior descamación al descender el nivel estrogénico. Esto último ocurre en la primera semana de vida, con un pico de aparición al quinto día. En general, dura de 3 días hasta un máximo de 3 semanas. ${ }^{6-7}$

Una vez involucionadas, en las niñas, las mamas permanecen en estadio de Tanner I hasta el comienzo del impacto estrógenico sobre ellas, situación que se produce alrededor de los 8 años de edad.

En la telorragia, la secreción a través del pezón es hemorrágica; se trata de una rara entidad en la población pediátrica cuya principal causa es la ectasia ductal benigna. Esta produce la dilatación de los conductos galactóforos de la región subareolar, con la distorsión de estos por fibrosis e inflamación. La dilatación origina una considerable desproporción con respecto a otros conductos, por lo que material lipídico y detritos ocupan su cavidad. Con la evolución, las células gigantes fagocitarias que rodean al material lipídico junto con los histiocitos forman un tejido de granulación con ulceración del epitelio ductal, que será responsable de la telorragia posterior. ${ }^{8}$

Suele presentarse fundamentalmente en los lactantes, pero se han informado casos prepuberales e, incluso, durante la pubertad (aunque en estos últimos deberían plantearse otros diagnósticos diferenciales incluidos los papilomas). ${ }^{2}$ Es más frecuente en los varones, con una relación 10:4. ${ }^{4}$

Hasta el momento no se conoce la causa y se han propuesto estímulos hormonales, anomalías estructurales del tejido mamario, infecciones $\mathrm{y}$, en un trabajo publicado recientemente, hematopoyesis extramedular. ${ }^{3}$

Para algunos autores el paso de hormonas por vía transplacentaria o a través de la leche materna podría estar implicado en el desarrollo de la ectasia. De esta forma los estrógenos generarían el crecimiento del sistema ductal y la progesterona el de los alvéolos mamarios. ${ }^{1-7}$ Esta hipótesis no ha sido demostrada y se han publicado casos tanto en lactantes alimentados con pecho como con fórmulas artificiales. En ninguno de los pacientes descritos habían aumentado los valores de estas hormonas.

En cuanto al diagnóstico y manejo clínico de los lactantes con telorragia, los datos aportados por la bibliografía se basan en informes de casos y recomendaciones de expertos. La ecografía mamaria, el estudio citológico de la secreción y, frente al aumento del tejido mamario, la medición de los niveles hormonales (prolactina, estradiol y hormonas tiroideas), suelen ser suficientes. ${ }^{1-5} \mathrm{La}$ ecografía puede evidenciar hallazgos compatibles o ser normal. En cuanto al estudio citológico, si bien su utilidad es limitada, permitiría reforzar la ausencia de células atípicas y de procesos inflamatorios agudos.

Se deben evitar los procedimientos invasivos, incluida la biopsia.

Con respecto a los diagnósticos diferenciales que podrían plantearse, detallamos a continuación algunos conceptos que nos parecen de importancia.

La mastitis en general se observa en lactantes de menos de 6 semanas de vida, es mayoritariamente unilateral, se acompaña de dolor y tumefacción en el área afectada y la secreción suele ser purulenta.

En los adenomas hipofisarios, y específicamente en los prolactinomas, hay aumento del tejido mamario generalmente bilateral, la secreción a través del pezón es galactorrea (no hemorrágica), puede acompañarse de cefalea, alteraciones visuales y amenorrea (esto último en mujeres con desarrollo puberal). ${ }^{5}$

Los diagnósticos de papiloma intraductal o de tumor phyllodes no deberían plantearse en los lactantes y los pacientes prepuberales teniendo en cuenta la epidemiología de estos tumores, y la ausencia de dolor o tumoración palpable. ${ }^{9}$ 
Con respecto a la terapéutica, hay consenso en adoptar una conducta expectante. No parece razonable la exéresis glandular, practicada en algunos informes, ya que la hemorragia se resuelve en forma espontánea habitualmente en pocas semanas, con un rango entre uno y 9 meses. ${ }^{4}$

En los últimos años, hemos tenido la oportunidad de observar en el Servicio de Bajo Riesgo del Hospital Garrahan a tres lactantes con características clínicas y evolución muy similar, en quienes la telorragia se resolvió en forma espontánea en un período de entre 4 y 12 semanas. El primero de ellos no fue documentado en su momento, por lo cual no se incluye en esta serie de casos.

En las tres oportunidades se trató de varones con un rango de edad comprendido entre 3 y 5 meses, y sin antecedentes de importancia. Los estudios hormonales y de laboratorio fueron normales, dos de ellos presentaron hallazgos ecográficos típicos de ectasia ductal y uno, ecografía normal.

\section{CONCLUSIONES}

Dado que, en los lactantes, la telorragia es un proceso benigno y autolimitado, sugerimos, en ausencia de otros hallazgos en el examen físico, realizar estudios mínimamente invasivos, adoptar una conducta expectante y tranquilizar a la familia en espera de la resolución espontánea.

\section{Agradecimientos}

Agradecemos al Dr. Esteban Dardanelli su colaboración con las imágenes de las ecografías presentadas.

\section{BIBLIOGRAFÍA}

1. Kelly VM, Arif K, Ralston S, Greger N, et al. Bloody nipple discharge in an infant and a proposed diagnostic. Pediatrics 2006;117:814-6.

2. Leung $\mathrm{AK}, \mathrm{Kao} \mathrm{CP}$. Mammary duct ectasia: a cause of bloody nipple discharge. J Nat Med Ass 2004;96(4):543-5.

3. Pampal A, Gokoz A, Sipahi T, Dogan H, et al. Bloody nipple discharge in 2 infants with interesting cytologic findings of extramedullary hematopoiesis and hemophagocytosis. J Pediatr Hematol Oncol 2012;34(3):229-31.

4. McHoney M, Munro F, Mackinlay G. Mammary duct ectasia in children: report of a short series and review of the literature. Early Hum Dev 2011;87(8):527-30.

5. Colao A, LocheS, Cappa M, DiSarno A, et al. Prolactinomas in children and adolescents: clinical presentation and longterm follow-up. J Clin Endocrinol Metab 1998;83:2777-80.

6. Lewitan de Eidelsztein, G. Ginecología de niñas y adolescentes: Diagnóstico, tratamiento y prevención. Capítulo 2. $1^{\text {a }}$ ed., Buenos Aires: Journal; 2013.

7. Manual de ginecología infantojuvenil.Sociedad Argentina de Ginecología Infanto Juvenil. Capitulo 14: Hemorragia genital en la niña. $2^{\mathrm{da}} \mathrm{ed}$. Ascune; 2003.

8. Plasencia Fernández E, Fontán Domínguez M, Rey Rey MJ, Miguez Silva F. Aproximación diagnóstica y manejo de la telorragia. Rev Pediatr Aten Primaria 2008;10:457-62.

9. Martínez Blanco J, Lombraña Álvarez E. Telorragia en la infancia, ¿motivo de alarma? Boletín de la sociedad de pediatría de Asturias, Cantabria Castilla y León. Volumen Pediátrico 2012; 52: 175-178. [Aceso: 1-10-13]. Disponible en: http://www.sccalp.org/documents/0000/1880/ BolPediatr2012_52_175_178.pdf 